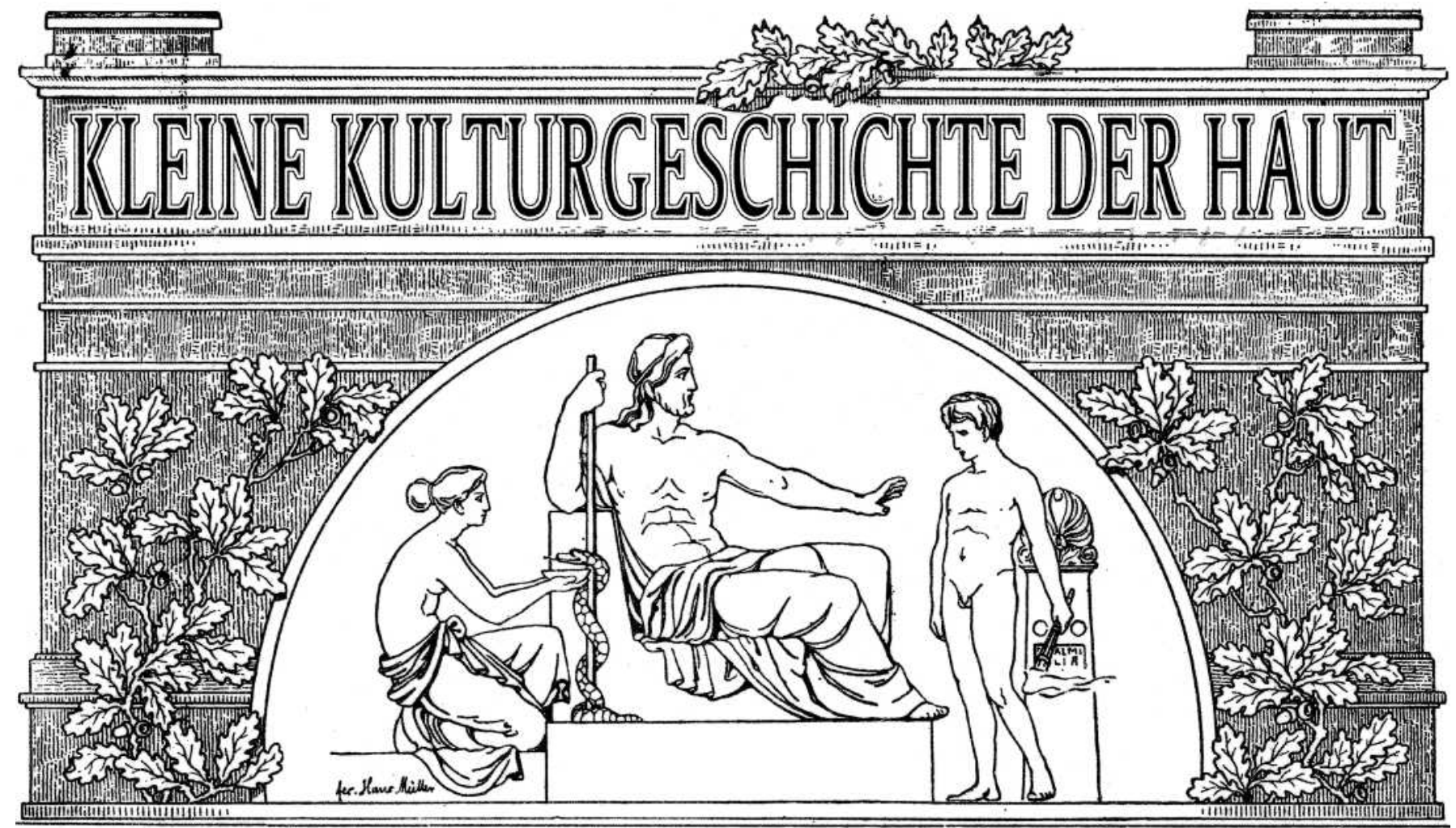

Die Zeitschrift „Aktuelle Dermatologie“ hat mit Beginn des Jahres 2004 diese Rubrik neu geöffnet, um in loser Folge kurze, prägnante und informative Artikel zu publizieren zu vielen Aspekten der Kulturgeschichte in Beziehung zur Haut. Dies erstreckt sich auf alle Kulturen und Zeiten, auf die Künste, auf Mythen und Psychologie und natürlich auch auf medizinische Aspekte.
Unsere Hoffnung, dem geneigten Leser Interessantes zu bieten, den Blick zu weiten und Freude zu bereiten, hat sich auf das Trefflichste bewährt. Reichlich Zusprüche, Kommentare, Vorschläge und aktive Teilnahme haben uns erreicht und erfreut. Dies möge weiter so gehen. Und so werden auch wir fleißig bemüht bleiben, Sie zu erbauen mit unserer kleinen Kulturgeschichte der Haut

\title{
Berührungen, Beziehungen
}

\section{U. Gerhardt}

Die Kultur des Menschen reicht bis in psychische und gesellschaftliche Zusammenhänge hinein. In die Kulturgeschichte der Haut gehören nicht nur Gebräuche der Vergangenheit, sondern auch Gegebenheiten der Gegenwart. Dabei können psychologische und soziologische Überlegungen eine Brücke bilden zum Verständnis sowohl körperlicher Zustände als auch kultureller Erscheinungen.

\section{Die Welt der ersten Lebensmonate}

Der aus Deutschland vertriebene Kinderarzt René Spitz, dessen klinische Beobachtungen an Kleinstkindern weltberühmt wurden, machte in den vierziger Jahren eine Entdeckung, die wohl bis heute gilt: Babys mit Ekzemen, so fand Spitz, hatten meistens Mütter, die es möglichst vermieden, ihre Kinder zu berühren. Diese Mütter scheuten sich also, ihre Babys anzufassen bzw. mit ihnen Hautkontakt zu haben. Spitz interpretierte den Befund psychologisch - und zwar aus der Warte des Säuglings. Die Babys, so seine These, ver- missten den Hautkontakt mit ihrer Mutter derart, dass sie durch die dermatologische Erkrankung gewissermaßen einen Anreiz für die Mutter bilden mochten, sich der Haut ihres Kindes zuzuwenden. Da die Mutter einen Arzt aufsuchte, kann man sicherlich sagen, dass die „List“ des Kindes (im vorsprachlichen Alter), seine Mutter auf seine Haut aufmerksam zu machen, durchaus erfolgreich zu nennen war, wenn die Mutter das Ekzem nach Anweisung des Arztes pflegte und dabei mit Cremes und Salben die Haut des Säuglings behandelte. Die Mütter, denen dieser Arzt den Zusammenhang zwischen dem Hautkontakt und dem dermatologischen Befund erläuterte, wandten sich jedenfalls offenbar ihren Babys mit nunmehr wissentlicher „Kontaktfreude“ zu - denn, wie Spitz akribisch notierte, die Ekzeme dieser Säuglinge verschwanden und traten auch späterhin nicht wieder auf.

Das Taktile, so folgerte Spitz aus diesen und anderen Beobachtungen, die er kinderpsychologisch interpretierte, hat einen hervorragenden Stellenwert für die Frühphase des Lebens. Die daraus hergeleitete These: In den ersten drei Lebensmonaten wird der Mensch durch zwei Arten von Sinneswahrnehmungen hauptsäch- 
lich geprägt. Das eine zentrale Sinnesorgan dieser Lebensphase ist der Tastsinn, also das Taktile, und das andere ist das Gehör, also der akustische Reiz. Niemals später im Leben habe das Taktile eine derart überwältigende Bedeutung für die Welterfahrung wie in den ersten Lebensmonaten [1]. Das Streicheln, Schmusen, Knuddeln, Liebkosen - wofür es Ausdrücke in allen Sprachen gibt wird in den ersten drei Lebensmonaten sozusagen Lebensnerv und Tor zur Welt. Beziehungsweise: Der Hautkontakt ist eine hauptsächliche Quelle der Lust des Seins in den ersten Monaten nach der Geburt.

Die kinderpsychologische These entspricht den durch Forschung untermauerten klinischen Erkenntnissen. Hirnphysiologisch lassen sich die Zusammenhänge zwischen frühkindlicher Entwicklung und der Haut als Schlüsselorgan plausibel explizieren. Die Beobachtungen und Thesen aus kinderpsychologischer Perspektive widersprechen den klinischen Tatsachen nicht.

Die Zeit der ersten Lebensmonate, so argumentierte Sigmund Freud vor genau einem Jahrhundert im Jahr 1906, ist eine Phase ungeteilt erotischer Lebenserfahrung. Erotik - Freud spricht hier von Sexualität in einem ganz anderen Sinn als im normalen Sprachgebrauch - ist im ersten Lebensabschnitt eine in die Welt hinein zerfließende Erfahrung des Verschmelzens und Einsseins. Im frühesten Lebensabschnitt, wo das Taktile - Berührung durch Hautkontakt - ein Lebenselixier ist, herrscht im Kleinstkind, so Freud, ein „ozeanisches Gefühl“ unendlicher Lustempfindung. Darin liege die Kraft des Lebenstriebes - dies ist der Sexualtrieb in seiner ursprünglichen Gestalt. Die Grunderfahrung des Menschen nach seiner Geburt ist Einssein, worin Freud die Urform des Sexuellen sieht. Erst im Laufe des weiteren Lebens - beginnend später im Säuglingsalter - werden die Lustempfindungen auf das Berühren besonderer Körperzonen konzentriert - und die so genannten erogenen Zonen sind ganz andere beim Kind als später bei Erwachsenen [2]. Das Taktile spielt allerdings nicht nur beim Säugling eine Hauptrolle. Das Kind im so genannten ödipalen Alter in einer schwärmerischen Liebe für den andersgeschlechtlichen Elternteil, der oder die Heranwachsende in der Pubertät und schließlich der oder die Erwachsene in ihrer Liebe (wofür Freud den Begriff Genitalität, nicht Sexualität, wählt) erleben jeweils anders und typisch für die verschiedenen Lebensphasen ihre eigene Lust an der Berührung. Jedenfalls ist die Haut allemal das Organ, das für Zärtlichkeit eine ganz besondere Empfänglichkeit hat.

\section{Die gesellschaftlichen Formen}

Von diesem Gesichtspunkt aus ist es nicht weit bis zu dem Gedanken, dass die Haut ein geselliges Organ ist. Das Gesellschaftliche in den Menschen und eben auch das Gesellschaftliche, das zwischen den Menschen geschieht, ist in vielerlei Weise mit Berührungen verbunden und wird durch Beziehungen symbolisiert.

Der Philosoph Georg Simmel, der um die vorige Jahrhundertwende unternahm, die Soziologie als eine geistes- und kulturwissenschaftliche Disziplin zu begründen, schrieb über die „höhere Einheit, die man ,Gesellschaft" nennt“ [3]. Man sehe die „unübersehbar mannigfaltigen Formen des sozialen Lebens, all das Miteinander, Füreinander, Ineinander, Gegeneinander, Durcheinander in Staat und Gemeinde, in Kirche und Wirtschaftsgenossenschaft, in

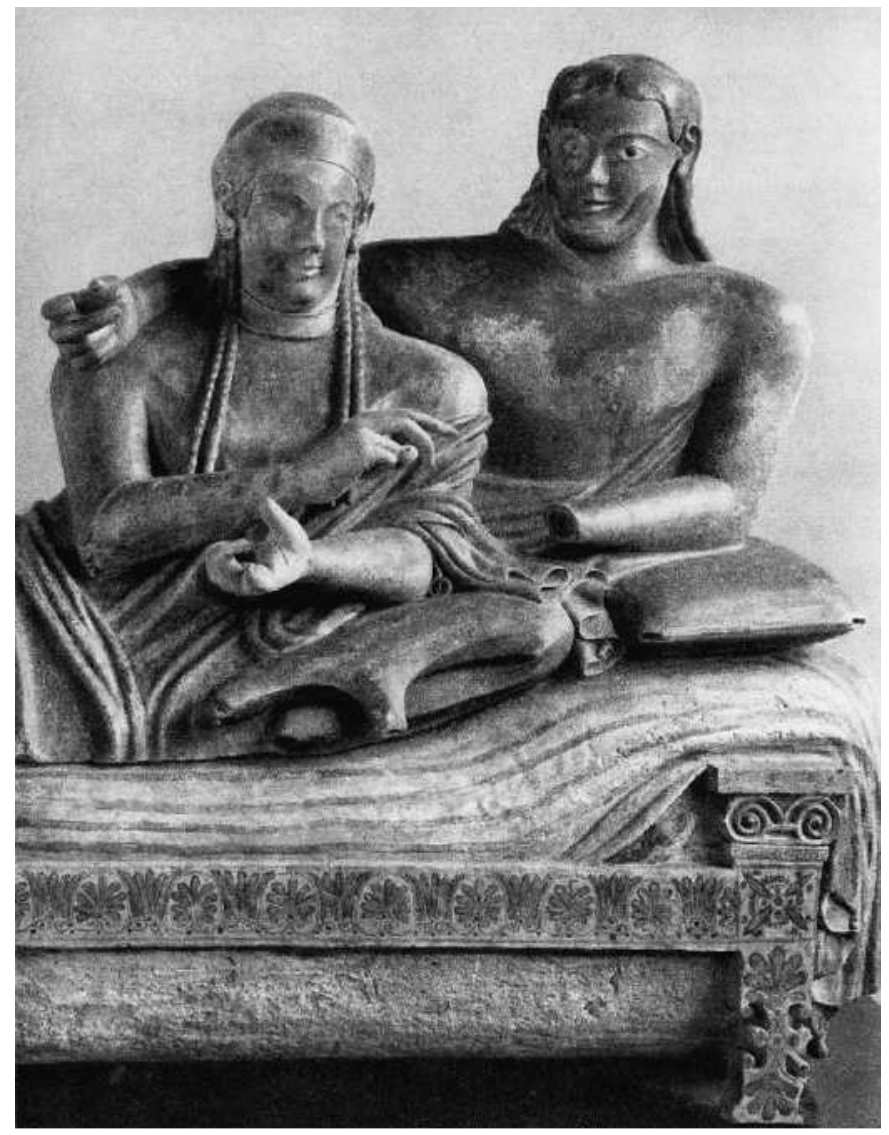

Abb. 1 Ein etruskischer Terrakottasarkophag aus der Nekropole von Caere (Ende 6. Jh. v. Chr., Paris, Louvre) versinnbildlicht die körperliche Vertrautheit der Ehepartner.

Familie und Vereinen ..., all die Vereinigungsformen, durch die aus einer bloßen Anzahl nebeneinander bestehender Wesen jedes Mal eine ,Gesellschaft‘ wird“.

In seinem zweiten Hauptwerk mit dem Titel Soziologie und dem Untertitel Untersuchungen über die Formen der Vergesellschaftung führte Simmel noch weiter aus, was dabei „Gesellschaft“ heißt [4]. Der andere Mensch, mit dem man allemal gesellschaftliche und zuweilen auch gesellige Kontakte pflegt (oder haben könnte), so Simmel, ist nicht schlichtweg ein Mensch als solcher, sondern, so Simmel im Weltgefühl des wilhelminischen Deutschland: „Der Bürgerliche, der einen Offizier kennenlernt, kann sich gar nicht davon freimachen, dass dieses Individuum ein Offizier ist... Und so geht es dem Protestanten gegenüber dem Katholiken, dem Kaufmann gegenüber dem Beamten, dem Laien gegenüber dem Priester usw.“

Bemerkenswert an den sozialen Beziehungen, die die Gesellschaft ausmachen, ist zweierlei. Zum einen sind soziale Beziehungen immer anders. Zwischen dem Geschäftsmann und dem Beamten herrschen beispielsweise ganz andere Verkehrsformen als etwa zwischen einem Gläubigen und einem Priester oder auch ganz gewiss zwischen Ehegatten, zwischen Eltern und Kindern und zudem zwischen Bekannten oder Freunden. Soziale Beziehungen enthalten normalerweise eine bestimmte Nähe oder Distanz im Umgang miteinander - und alle Angehörigen einer Kultur oder Gesellschaft sind sich bewusst, dass bestimmte Beziehungen eher 
unpersönlich oder eher persönlich sind, also mehr Distanz oder mehr Nähe bedeuten. Zum anderen sind mit diesem „Füreinander, Ineinander, Gegeneinander“, wie Simmel es nennt, ganz bestimmte Verhaltensweisen gemeint (Abb.1). Typischerweise und erlaubtermaßen kann oder darf man bestimmte Dinge tun oder nicht tun woraus sich Rückschlüsse auf die entsprechenden Beziehungen ergeben (können). Ein Beispiel: Wenn Eheleute sich allenfalls die Hände reichen und ansonsten keinen weiteren Körperkontakt haben, wird man daraus schließen, dass ihre Ehebeziehung zumindest ungewöhnlich ist; wenn ein Geschäftsmann und ein Beamter (etwa eines Finanzamts) sich zur Begrüßung die Hand reichen und keinen weiteren Körperkontakt haben, tun sie, was man gewöhnlich in einer solchen sozialen Beziehung tut.

Mit anderen Worten: Die „Gesellschaft“, also die gesellschaftliche Welt, umfasst Regeln über Nähe und Distanz zu den anderen Angehörigen derselben Kultur oder Gesellschaft. Dabei ist der Körperkontakt, die körperliche Berührung, kulturell normiert. Die Normen, die unsere körperlichen Berührungen - in der Öffentlichkeit, im Privaten - regeln, können weit gefasst sein und dabei eine gewisse Toleranz für Individualität erlauben. Oder diese Normen können relativ streng vorgeben, was erlaubt oder verboten ist. Die Spielbreite und die Strenge der entsprechenden Normen kann sich zudem im Laufe der Zeit wandeln.

Offenbar enthält das „Füreinander, Ineinander, Gegeneinander“ der Menschen in der Gesellschaft - mit Ausnahmen, wofür wiederum Regeln existieren - auch kulturelle Regelungen des EinanderBerührens. So ist das Sich-Umarmen oder Händeschütteln oder Handgeben kulturell - mit dabei durchaus variablen Toleranzspielräumen - per Etikette etc. vorgegeben. Für unterschiedliche Kulturen gilt dasselbe Verhalten zuweilen anders. Die Begrüßung per Handschlag symbolisiert etwas anderes in der angelsächsischen Welt als in Deutschland, und Bruderschaftsküsse zur Begrüßung bedeuten etwas anderes in Russland oder Frankreich als in Deutschland. Eskimos, so sagt man, begrüßen einander, indem sie ihre Nasen aneinander reiben - was dieselbe Beziehung signalisiert, die in Mitteleuropa vielleicht ein freundschaftlicher Schlag auf die Schulter ausdrückt. Und außerdem gibt es einen gesellschaftlichen Wandel in den Berührungsge- und -verboten einer jeweiligen Gegenwart: Während es zur Nazizeit wohl allenfalls in einer Privatwohnung unproblematisch war, einen Menschen, der Jude war, zu umarmen, würde heute niemand an einer Umarmung auf der Straße Anstoß nehmen (und erst recht nicht die Polizei darüber informieren). Die Tagebücher des Romanisten Viktor Klemperer, der über die Alltagsdinge im Nationalsozialismus Zeugnis ablegte, das auch die Berührungen und die Beziehungen der Menschen damals schilderte, sind voller Szenen, die dies den Heutigen in Erinnerung rufen oder wieder bewusst machen [5].

\section{Die moderne Alltagskultur}

In der heutigen Welt gibt es Berührungen und Beziehungen im Alltag zwischen Personen, die einander nicht kennen und sich nie kennen lernen werden. Film und Fernsehen, Zeitschriften und Literatur bieten dem modernen Menschen Erlebnisse, die ihn oder sie in seiner oder ihrer Seele berühren und doch keine Beziehung zu einem Gegenüber in persona beinhalten. Die Kunst, der Sport und überhaupt die Welt der Schönen und Reichen schafften Beziehungen zwischen dem Einzelnen - als Kenner, Sportfan, Normalbür- ger, Teenager - und Persönlichkeiten der ganzen Welt. Berührung und Beziehung werden in dieser Welt der alltäglichen modernen Kultur möglich durch Identifikation mit einem Künstler, Könner oder Gönner. Berührung und Beziehung kann es sogar mit Kinohelden wie James Bond oder Bonny and Clyde geben - nicht zu vergessen die Lust am Berühren, die vielleicht in dem Gruseln mitschwingt, wenn King Kong sich im Urwald und in New York der schönen ohnmächtigen Blonden widmet.

Indirektes Erleben gehört zur modernen Welt der Gegenwart mindestens seit dem achtzehnten Jahrhundert, als Goethes Die Leiden des jungen Werther manche Leser zu Selbstmord verleitete [6]. Ganz sicherlich können hirnphysiologische Vorgänge erklären, wieso die Berührung, auch wenn physisch nichts geschieht, erlebt werden kann. Und kognitive Vorgänge können verständlich machen, dass gesellschaftliche Beziehungen gewissermaßen virtuell bestehen können. Jedenfalls lösen sie Begeisterung und Schmerz, Jubel und Trauer aus - was wohl im Juni dieses Jahres unübersehbar sein wird, wenn die Fußballweltmeisterschaft in Deutschland (fast) die ganze Nation mitreißt und Millionen Menschen in allen Teilen der Erde in ihren Bann zieht.

\section{Literatur}

${ }^{1}$ Spitz R. Die Entstehung der ersten Objektbeziehungen (Übersetzung aus dem Französischen: La première année de la vie de l'enfant, Paris 1945). Mit einem Geleitwort von Anna Freud. 2. Auflage. Stuttgart: 1960

2 Freud S. Drei Abhandlungen zur Sexualtheorie (ursprünglich 1906). Gesammelte Schriften, Bd. V. London: 1942: 27-145

${ }^{3}$ Simmel G. Soziologie der Geselligkeit (ursprünglich 1911). Georg Simmel Gesamtausgabe, Bd. 12: Aufsätze und Abhandlungen 1909-1918. Frankfurt: 2001: 177-193

${ }^{4}$ Simmel G. Soziologie. Untersuchungen über die Formen der Vergesellschaftung (ursprünglich 1908). Georg Simmel Gesamtausgabe, Bd. 11. Frankfurt: 1992

${ }^{5}$ Klemperer V. Ich will Zeugnis ablegen bis zum letzten. Band I: Tagebücher 1933 -1941, Band II: Tagebücher 1942 -1945. 4. Auflage. Berlin: 1995

${ }^{6}$ Gerhardt U. Gesellschaft und Gesundheit. Begründung der Medizinsoziologie. Frankfurt: 1991

Prof. Dr. Uta Gerhardt

Lehrstuhl für Soziologie II

Universität Heidelberg

Sandgasse 7-9

69117 Heidelberg

E-mail: uta.gerhardt@urz.uni-heidelberg.de 